\title{
Application of communication systems via the ultraviolet channel in FANET networks
}

\author{
Gleb S. Vasilyev ${ }^{1, *}$, Oleg R. Kuzichkin ${ }^{1}$, Dmitriy I. Surzhik ${ }^{2}$, and vetlana M. Kharchuk ${ }^{2}$ \\ ${ }^{1}$ Belgorod National Research University, 85 Pobedy St., 308015 Belgorod, Russia \\ ${ }^{2}$ Vladimir State University, 87 Gorky St., 600000, Vladimir, Russia.
}

Keywords: Unmanned aerial vehicle, UAV, Flying ad-hoc network, FANET, Physical layer, Ultraviolet communication, UV-C, NLOS, DPIM modulation.

\begin{abstract}
The possibility of providing communication in difficult conditions on the basis of Flying Ad Hoc Networks (FANET) requires the use of non-electronic means of communication. It is proposed to use optical communication in the UV-C range in the absence of line-of-sight between the nodes of the FANET network. The general scheme of the optical transmitter and receiver of UV-C range is considered and the review of the components required for its implementation, taking into account the application possibility in highly mobile small-sized UAVs, is carried out. As a result of the analysis of FANET networks with UV-C channel, physical and channel level parameters critical for network performance are revealed. Methods of adaptive change of these parameters in the practical implementation of the UV-C communication system in FANET networks are proposed.
\end{abstract}

\section{Introduction}

The capabilities of existing intelligent decision-making systems are very limited, so for the successful solution of a wide class of tasks based on unmanned aerial vehicles (UAVs) reliable communication with the group of aircraft is required [1]. For communication between individual UAVs, as well as UAVs with a ground station, Flying Ad Hoc Networks (FANET) and flying sensor networks (FSN) are used. Known works devoted to the development and research of such networks consider predominantly radio communication at the physical level [2,3]. At the same time, there may be situations when radio communication is ineffective or impossible:

1) the presence of high obstacles in dense urban areas or difficult terrain;

2) high level of external electromagnetic (EM) background, which is typical for modern cities and many technical facilities;

3 ) temporary failure of radio communication systems as a result of electronic warfare;

4) destruction of existing communications infrastructure as a result of an emergency.

\footnotetext{
* Corresponding author: vasilievgleb@yandex.ru
} 
The use of optical communication systems that are not affected by EM interference is an effective solution in a complex EM environment. However, optical communication systems in the infrared range (IrDA) [4,5] have an extremely small distance (units of meters), unacceptable for communication with UAVs. In addition, infrared communication works only in line-of-sight (LOS) conditions and is not applicable in the presence of obstacles. Optical visible light communication (VLC) systems [6-8] have a range sufficient to communicate with UAVs (more than $1 \mathrm{~km}$ ), but also operate only in LOS mode.

Communication systems of the sun-blind ultraviolet range UV-C from 200 to $280 \mathrm{~nm}$ provide the possibility of communication in the non line-of-sight mode (NLOS) due to the strong scattering of UV radiation in the atmosphere, the communication range is up to 2-5 $\mathrm{km}$ [9]. Thus, only this method of communication is effective in complex conditions mentioned above (1)-(4). Existing studies consider the use of UV-C communication systems between stationary objects [9-12] or mobile nodes [13] without taking into account the specifics of their application in aircraft networks.

The aim of the work is to substantiate the use of communication systems via the ultraviolet channel in FANET networks.

\section{Main part}

\subsection{Geometry of NLOS UV communication channel with UAV}

The general scheme of the communication channel with the UAV group is shown in Fig. 1. In this topology, each slave UAV (for example, UAV 1) transmits its data to the main UAV $\mathrm{K}$, and it, in turn, transmits information to the ground base station.

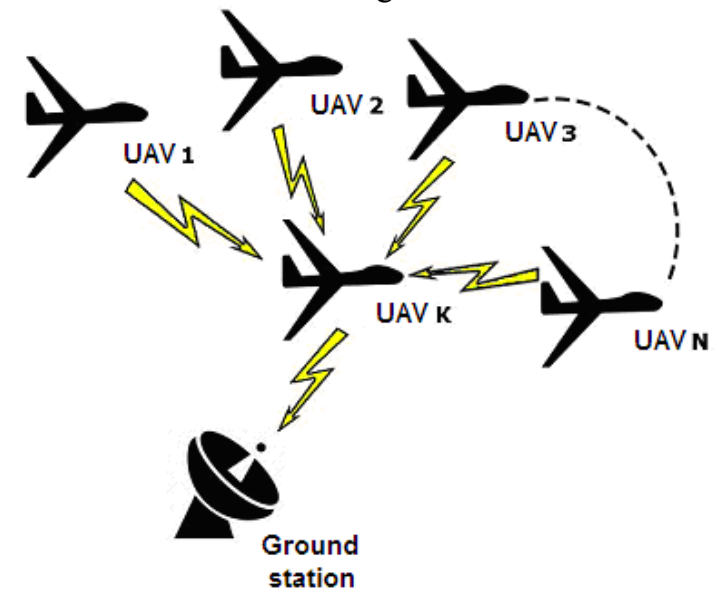

Fig.1. Scheme of communication channel.

Due to the presence of terrain obstacles or high-rise buildings, direct visibility between communication nodes in a "UAV-UAV" or "UAV - ground station" type scenario may not be available. Communication channel geometry in the NLOS mode due to radiation scattering in the atmosphere is shown in Fig. 2. Fig. 2A shows the vertical projection of the channel and denotes: Tx - ground station transmitter, $\mathrm{Rx}$ - UAV receiver, $\mathrm{r}$ - the distance between $\mathrm{Tx}$ and $\mathrm{Rx}, \theta 1,2$ and $\varphi 1,2$ - an elevation and width angle of the radiation pattern, index 1 refers to the transmitter, index 2 -to the receiver, $\theta$ s is a scattering angle, $\mathrm{V}$ - the total volume of the Tx and Rx radiation patterns, $\mathrm{r} 1,2$ - the distance from Tx and Rx to the center of the area $\mathrm{V}$, the gray rectangle designates an obstacle. 
To analyze the performance of a network with different spatial orientations of network nodes, it is also necessary to take into account the azimuths of the transmitter and receiver $\psi \mathrm{T}, \mathrm{R}$, as shown in Fig. $2 \mathrm{~B}$.
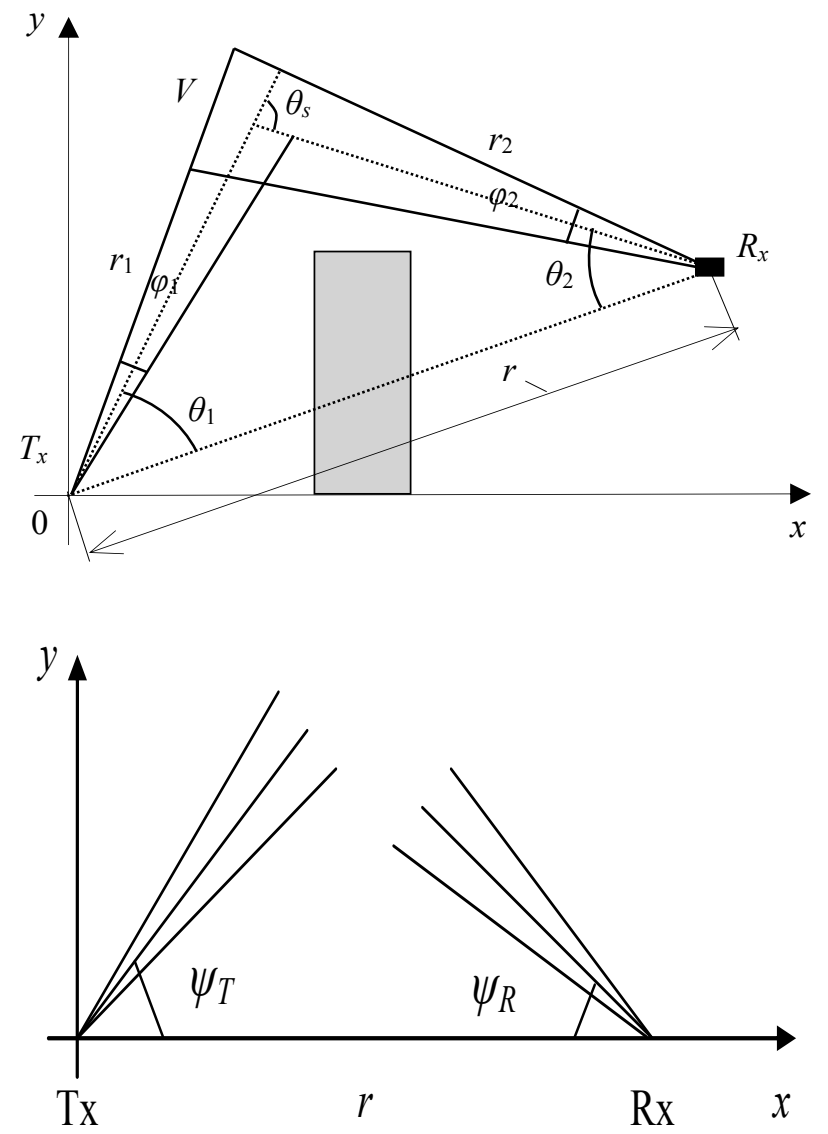

Figure 2. Vertical (A) and horizontal (B) UV channel projection at line-of-sight (NLOS) mode with one transmitter and one receiver

\subsection{Physical implementation of UV-C communication system for use in flying networks}

The general scheme of the single-channel UV communication system is shown in Fig. 3.

UV lasers [10,11], single LEDs or arrays of LEDs [14,15] are used as optical emitters in UV communication systems. UV-C lasers have substantially more radiated power than UVC LEDs: a maximum of about $1 \mathrm{~W}$ for lasers versus 130mw for LEDs (for example, SMD 6060 UVC LED High-Power). Despite the lower radiation power by an order of magnitude, the use of LEDs in the onboard transmitter of a small-sized UAV is more relevant than lasers, which require a complex and cumbersome charge-pumping system. In addition, it is possible to use LED matrices with radiated power up to $2 \mathrm{~W}$ (for example, FLS 6060 UVC SMD LED 5x5 Array). 


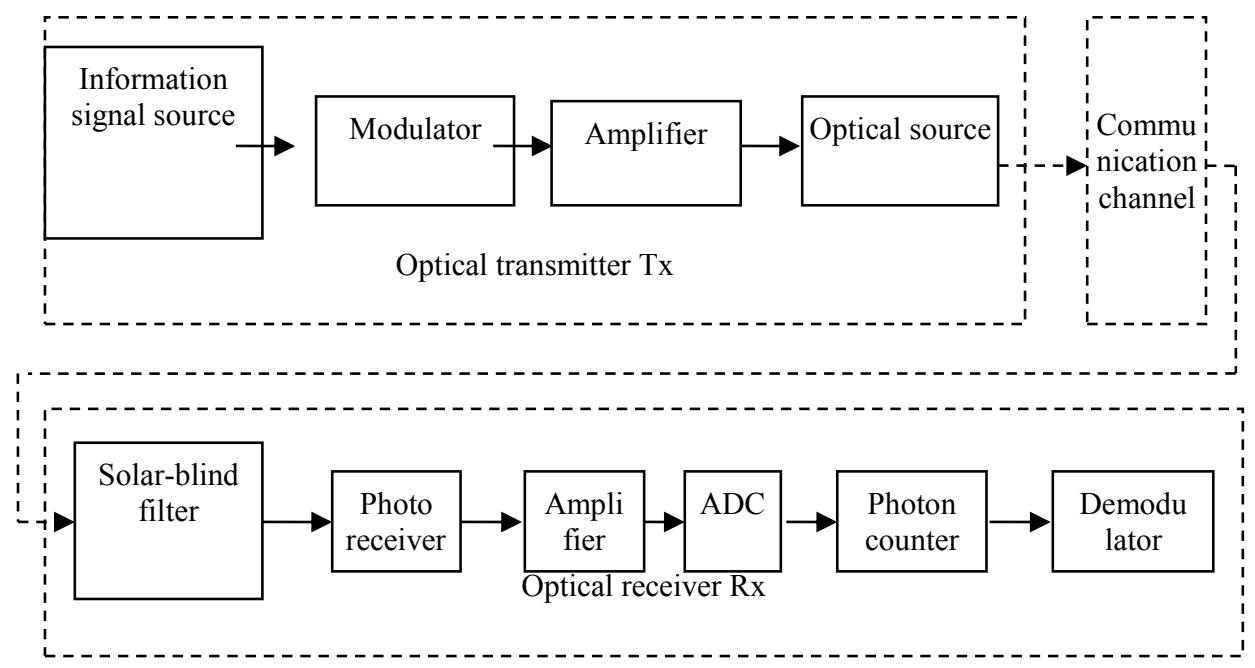

Fig. 3. General scheme of a single-channel UV-C communication system.

As a photodetector, it is advisable to use a photoelectronic multiplier tube (PMT), since the alternative - a photodiode or an array of photodiodes - does not have sufficient sensitivity to receive a scattered signal in the NLOS mode [16]. Given the fragility of conventional PMT, it is advisable to consider the choice of ruggedized PMT for use on UAV board $[17,18]$.

An absorption-type solar-blind filter at the output of the PMT is required to block solar radiation with suppression of the visible range of more than 12 orders of magnitude [19]. A transimpedance amplifier is used to amplify the photodetector current [20]. The UV NLOS communication system must register single photons, so the analog mode of the PMT is not applicable, and it is necessary to use an ADC and a photon counter.

\subsection{Physical layer of flying networks with UV-C communication channel}

It should be noted that in UV communication systems, on-off keying (OOK) modulation is used most often, since its technical implementation is simpler compared to pulse-position modulation (PPM). At the same time, PPM modulation has higher energy efficiency, but it is based on the symbolic synchronization of the transmitter and receiver. Digital pulse interval modulation (DPIM) and dual-head DPIM (DH-DPIM) can modulate the same number of bits per character as even-order PPM, but does not require character synchronization. It is also important that DPIM and DH-DPIM use fewer time slots per symbol than PPM and thus require less bandwidth [22-26]. Like PPM, interval types of modulation have greater energy efficiency compared to conventional ones. These advantages determine the relevance of using DPIM and DH-DPIM in various UV communication systems.

These features are especially important for the implementation of the FANET network with UV channel, because a large number of subscribers in a limited space use a significant frequency resource. Approaches and solutions for frequency channel separation, as well as synchronization of the transmitter and receiver of UV radiation based on an external optical sync signal, unlike the radio frequency range, have not been developed. In known experiments, an additional radio frequency sync signal was used to synchronize the UV communication system. An external radio signal for synchronization may not be available due to obstacles or radio interference. Therefore, the use of self-synchronizing modulation and coding methods that economically use the available bandwidth, in particular DPIM and DH-DPIM, for flying UV communication networks is highly desirable. Time diagrams of 4- 
position pulse modulation PPM and DPIM with the number of guard intervals $n=1$ are shown in Fig. 4.

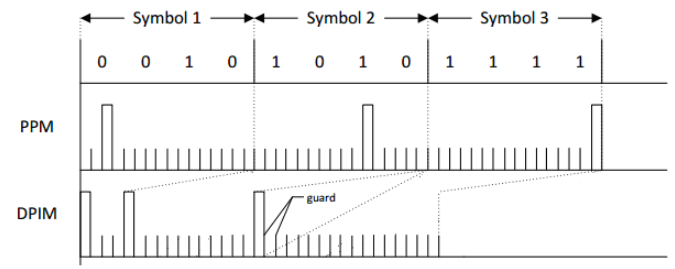

Fig. 4. Time diagrams of modulations 4-PPM and 4-DPIM, number of guard intervals $n=1$.

The energy characteristics of the L-position DPIM modulation $\left(L=2^{M}\right)$ with $n$ protection intervals are determined by the expression [27]:

$$
B E R_{\mathrm{DPIM}}(S N R)=\frac{1}{2} \operatorname{erfc}\left(\frac{1}{2 \sqrt{2}} \sqrt{S N R \cdot \frac{L}{2} \frac{L+2 n+1}{2 L} \log _{2} L}\right)
$$

where $\operatorname{erfc}(x)=\frac{2}{\sqrt{\pi}} \int_{x}^{\infty} \exp \left(-t^{2}\right) d t$ is the error function (probability integral).

The signal-to-noise ratio (SNR) of a UV channel is defined as the ratio of the number of detected signal photons $N_{d}$ to the number of noise photons $N_{n}$ :

$$
S N R=N_{d} / N_{n} 0
$$

where $N_{d}=\eta_{f} \eta_{r} N_{r}=\eta_{f} \eta_{r} N_{r} /$ Loss $=\eta_{f} \eta_{r} P_{t} \lambda /(h c R \cdot$ Loss $)$. Here it is indicated: $P_{t}$ radiation power of the transmitter, $\eta_{f}$ - transmission coefficient of the solar-blind filter, $\eta_{r}$ quantum efficiency of the detector (receiver), $N_{r}$ - the number of received photons, $\lambda-$ wavelength of radiation, Loss - losses in the channel, $R$ - bitrate, $h=6.626 \times 10^{-34} \mathrm{~J} * \mathrm{~s}-$ Planck constant, $c=3 \times 10^{8} \mathrm{~m} / \mathrm{s}$ - the speed of light in a vacuum. The results of the measurements showed that in the worst case, when the photodetector is directed to the sun in clear weather, the detection frequency of noise photons is about $15,000 \mathrm{~Hz}$ (using a highquality solar-blind filter and a receiver with aperture area less than $2 \mathrm{~cm}^{2}$ ) [14].

The loss value is determined by the channel parameters: communication range, elevation angles and azimuths of the transmitter and receiver, the width of their radiation patterns. In [13], the Monte Carlo method is used to simulate the magnitude of losses in the UV channel. The following values of the UV channel parameters were adopted: communication range $r$ $=100 \mathrm{~m}$, elevation angles of the transmitter and receiver $\theta_{1}=30^{\circ}$ and $\theta_{2}=50^{\circ}$, radiation pattern width of the transmitter and receiver $\varphi_{1}=17^{\circ}$ and $\varphi_{2}=30^{\circ}$, radiation wavelength $\lambda=260 \mathrm{~nm}$, scattering and absorption coefficients for clear weather, the aperture area of the receiver $A_{r}=1.77 \mathrm{~cm}^{2}$. The result is a parametric approximation models for the UV channel:

$$
\begin{gathered}
\operatorname{Loss}(r)=\xi r^{\alpha} e^{\beta r} \mathrm{~dB}, \xi=93 \mathrm{~dB}, \alpha=0.045, \beta=1.3 \cdot 10^{-5} ; \\
\operatorname{Loss}\left(\theta_{1}\right)=115-7.2 \cdot \exp \left(-0.031 \theta_{1}\right) \mathrm{dB} ; \\
\operatorname{Loss}\left(\theta_{2}\right)=113-3.1 \cdot \exp \left\{-0.032\left(\theta_{2}-20^{0}\right)\right\} \mathrm{dB} \\
\operatorname{Loss}\left(\phi_{2}\right)=107+14.9 \cdot \exp \left(-0.032 \phi_{2}\right) \mathrm{dB} \\
\operatorname{Loss}\left(\psi_{1}\right)=114+0.082 \cdot \psi_{1}^{2} \mathrm{~dB}, \psi 1=(-200 ; 200) ; \\
\operatorname{Loss}\left(\psi_{2}\right)=L_{0}+K \cdot \psi_{2}^{2} \mathrm{~dB}, \mathrm{~L} 0=114 \mathrm{~dB}, \mathrm{~K}=0.015, \psi 2=(-400 ; 400) ;
\end{gathered}
$$


Obtained parametric models have acceptable accuracy (less than $1.5 \mathrm{~dB}$ ) in a wide range of parameters of channel geometry, which allows using the obtained results for calculation and parameter optimization of wireless ad-hoc networks with UV channel in the process of setting up the transmission mode.

Based on the expressions (1)-(3), the energy characteristics of the UV communication system with 4-position DPIM modulation with a different number of guard intervals $(n=1,2$, 3) at a high noise level are as shown on Fig. 5 and Fig. 6.

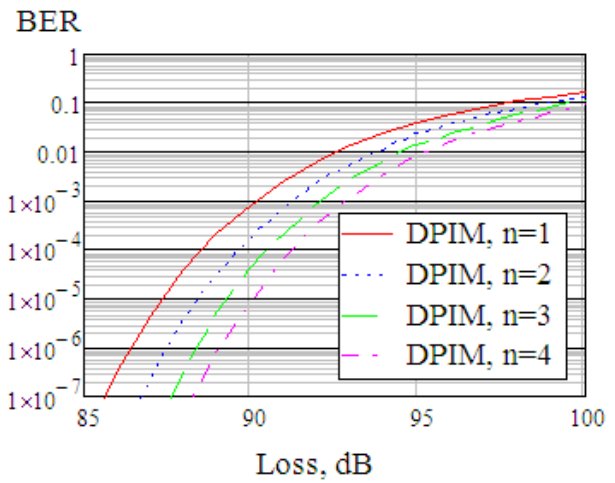

Fig. 5. Dependence of BER on losses for 4-DPIM modulation with different number of guard intervals (guard intervals $n=1,2,3,4$ ) at high noise level.

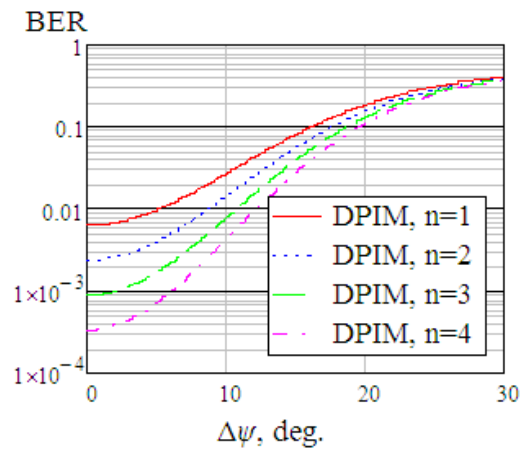

Fig. 6. Dependences of BER on azimuthal deviation $\Delta \psi$ for 4-DPIM modulation with different number of guard intervals $(n=1,2,3,4)$ at high noise level.

From Fig. 5 and 6 it can be seen that the noise immunity of the communication system with DPIM modulation can be increased by introducing additional protective intervals, which is accompanied by a decrease in the bit error rate. However, this is accompanied by a decrease in the bit rate of data transfer. This aspect determines the relevance of adaptive selection of the number of protective intervals in UV communication systems with DPIM modulation. An increase in the azimuthal deviation of the transmitter and receiver leads to an increase in losses and, respectively, BER at the same bit rate (Fig. 7), which makes it necessary to create guidance systems in the azimuthal plane. It is also promising to use a group of emitters directed at different azimuthal angles, and the inclusion of one of them to ensure the maximum signal level in specific conditions. 


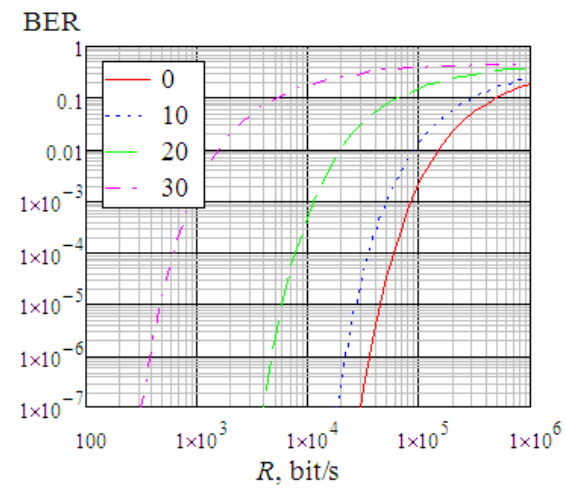

Fig. 7. - BER dependences for 4-DPIM modulation with two guard intervals $(n=2)$ at high noise level, azimuthal deviation $\Delta \psi=0 \ldots 30$ degrees.

According to the results of modeling (3) and experimental studies [9], the losses in the NLOS UV channel at ranges of the order of kilometers, relevant for the network of aircraft in urban conditions, are up to $160 \mathrm{~dB}$ or more. At the same time, at short distances (ones-tens of meters) losses are significantly less (less than $90 \mathrm{~dB}$ ). With a fixed transmitter power, such a large spread at the level of the received signal (more than $70 \mathrm{~dB}$ ) imposes difficult requirements for the depth and speed of adjustment of the receiver's automatic gain control system (fast adjustment is required due to the high mobility of communication nodes UAVs). Therefore, it is necessary to change the transmitter power adaptively depending on the specific conditions. According to (2), radiation power sufficient to achieve a given SNR value is determined by the expression:

$$
P_{t}\left(r, \theta_{1}, \theta_{2}, \psi_{1}, \psi_{2}\right)=\frac{S N R \cdot N_{n} \cdot h c R \cdot \operatorname{Loss}\left(r, \theta_{1}, \theta_{2}, \psi_{1}, \psi_{2}\right)}{\eta_{f} \eta_{r} \lambda}
$$

The radiated power of UV-C sources is limited to $1-2 \mathrm{~W}$, so when the maximum power value is exceeded, the condition (4) at a given bit rate R is not feasible, and it is necessary to reduce the bit rate:

$$
R=\frac{P_{\max } \cdot \eta_{f} \eta_{r} \lambda}{S N R \cdot N_{n} \cdot h c R \cdot \operatorname{Loss}\left(r, \theta_{1}, \theta_{2}, \psi_{1}, \psi_{2}\right)}, P_{t}\left(r, \theta_{1}, \theta_{2}, \psi_{1}, \psi_{2}\right)>P_{\max }
$$

\section{Conclusion}

The possibility of complex conditions, when radio communication is ineffective or impossible, forces the development and research of non-electronic means of communication for the organization of flying networks at the physical level. It is proved that for the organization of communication in the absence of line-of-sight between the nodes of the FANET network, communication in the infrared and visible range is not applicable, and it is necessary to use communication via the UV-C channel. The general scheme of the optical transmitter and receiver of UV-C range is considered and the review of the components required for its implementation, taking into account the possibility of application in highly mobile small-sized UAVs, is carried out. As a result of the analysis of the physical layer of FANET networks with UV-C communication channel, it was found that the digital pulse interval modulation DPIM and DH-DPIM has high energy efficiency and does not require 
external synchronization (such synchronization is not designed yet in the UV-C range). It is shown that in order to implement a practical UV-C communication system in FANET networks at the physical and channel level, it is necessary to implement an adaptive change of the following parameters:

- the number of guard intervals of interval modulation;

- orientation of emitters and receivers in the azimuthal plane, as well as in the vertical plane to avoid obstacles;

- power and bit rate.

The work was supported by RFBR grant 19-29-06030-MK "Research and development of wireless adhoc network technology between UAVs and control centers of "smart city" on the basis of adaptation of transmission mode parameters at different levels of network interaction"

\section{References}

1. R. Austin. Unmanned aircraft systems: UAVS Design, Development and Deployment / John Wiley \& Sons, Ltd., 2010.

2. J. Yin, G. Holl, T. Elbatt, F. Bai, H. Krishnan, DSRC channel fading analysis from empirical measurement, in: Proceedings of the 1st IEEE International Workshop on Vehicle Communications and Applications (Vehiclecomm), 2006, pp. 25-27.

3. I.Y. Abualhaol, M.M. Matalgah, Performance analysis of cooperative multi-carrier relay-based UAV networks over generalized fading channels, International Journal of Communication Systems 24 (8) (2011) 1049-1064.

4. Al-Nassar, Suha \& Hatem, Haraa \& Shehab, Jinan. (2018). Design and Implementation of Infrared (IR) Communication System. 29-33. 10.26367/DJES/VOL.11/NO.3/5.

5. Arvin, Farshad \& Samsudin, Khairulmizam \& Ramli, Abdul. (2009). A Short-Range Infrared Communication for Swarm Mobile Robots. Signal Processing Systems, International Conference on. 454-458. 10.1109/ICSPS.2009.88.

6. Ndjiongue, Alain Richard \& Ferreira, Hendrik \& Ngatched, Telex. (2015). Visible Light Communications (VLC) Technology. Wiley Encyclopedia of Electrical and Electronics Engineering. 1-15. 10.1002/047134608X.W8267.

7. Giustiniano, Domenico; Tippenhauer, Nils Ole; Mangold, Stefan (2012). "Lowcomplexity Visible Light Networking with LED-to-LED communication". 2012 IFIP Wireless Days. pp. 1-8. doi:10.1109/WD.2012.6402861. ISBN 978-1-4673-4404-3.

8. IEEE 802.15 WPAN Task Group 7 (TG7) Visible Light Communication

9. Chen G, Liao L, Li Z, et al. Experimental and simulated evaluation of long distance NLOS UV communication[C]//Communication Systems, Networks \& Digital Signal Processing (CSND-SP), 2014 9th International Symposium on. IEEE, 2014: 904-909.

10. $\mathrm{Xu} \mathrm{Z}$. and Sadler B. Ultraviolet communications: potential and state-of-the-art IEEE Commun. Mag.4667-73, 2009.

11. Han D, Liu Y, Zhang K, et al. Theoretical and experimental research on diversity reception technology in NLOS UV communication system[J]. Optics express, 2012, 20(14): 15833-15842.

12. Shaw G., Nischan M., Iyengar M., Kaushik S. and Griffin M. NLOS UV communication for distributed sensor systems Proc. SPIE412683-96, 2000.

13. Konstantinov I.S., Vasilyev G.S., Kuzichkin O.R., Surzhik D.I., Lazarev S.A.. Numerical and Analytical Modeling of Wireless UV Communication Channels for the Organization of Wireless Ad-Hoc Network // IJCSNS - International Journal of 
Computer Science and Network Security - 2018. - Vol. 18, No. 8, pp. 98-104. Open access: http://paper.ijcsns.org/07_book/201808/20180815.pdf.

14. Chen G, Abou-Galala F, Xu Z, et al. Experimental evaluation of LED-based solar blind NLOS communication links[J]. Optics Express, 2008, 16(19): 15059-15068

15. Guo Q, He N, He Z. Research on the channel performances and transmission in UVLED scatter communications[J]. Study On Optical Com-munications, 2013 (3): 64-66

16. Raptis, Nikos \& Pikasis, Evangelos \& Syvridis, D.. (2016). Performance evaluation of non-line-of-sight optical communication system operating in the solar-blind ultraviolet spectrum. 999107. 10.1117/12.2241424.

17. Hamamatsu Photomultiplier Tubes: basics and applications. - 4th edition, - P. 258.

18. Bicron Corp.: Ruggedized High-Temperature Detector Technology.

19. Ofil's Solar blind UV filters: http://www.sbuv.com/filters/sb268.html.

20. Eduard Säckinger. Analysis and Design of Transimpedance Amplifiers for Optical Receivers, 2007, 592 p, ISBN: 978-1-119-26441-5.

21. Xu C. and Zhang H., "Packet error rate analysis of IM/DD systems for ultraviolet scattering communications", IEEE Military Communications Conference (2015), pp. 1188-1193.

22. Ghassemlooy Z., Kaluarachchi E. D., Reyher R. U., and Simmonds A. J., "A new modulation technique based on digital pulse interval modulation (DPIM) for opticalfiber communication,” Microw. Opt. Technol. Lett., vol. 10, no. 1, pp. 1-4, Sep. 1995.

23. Ghassemlooy Z., and Hayes A. R., "Digital pulse interval modulation for optical communications,” IEEE Commun. Mag., vol. 36, no. 12, pp. 95-99, Dec. 1998.

24. Hu Z., Tang J., "Performance of digital pulse interval modulation of atmospheric optical wireless communication system," Proc. SPIE, vol. 5625, pp. 202-208, Feb. 2005.

25. Aldibbiat N. M., Ghassemlooy Z., and McLaughlin R., "Error performance of dual header pulse interval modulation (DH-PIM) in optical wireless communications," IEE Proc. Optoelectron., vol. 148, no. 2, pp. 91-96, Apr. 2001.

26. Ma J., Jiang Y., Yu S., Tan L., and Du W., "Packet error rate analysis of OOK, DPIM and PPM modulation schemes for ground-to-satellite optical communications," Opt. Commun., vol. 283, pp. 237-242, Jan. 2010.

27. Igor S. Konstantinov, G. S. Vasyliev, Oleg R. Kuzichkin, D. I. Surzhik, I. A. Kurilov, Sergey A. Lazarev. AUV Link Mobile Ad-Hoc Network Examination, International Journal of Engineering and Advanced Technology (IJEAT) ISSN: 2249 - 8958, Volume-8, Issue-5S July 2019, DOI:10.35940/ijeat.E1063.0785S319.

28. I.S. Konstantinov, G.S. Vasilyev, O.R. Kuzichkin, I.A. Kurilov, S.A. Lazarev. Modeling and Analysis of the Characteristics of Ultraviolet Channels under Different Conditions of Radiation Propagation for the Organization of Wireless AD-HOC Network // JARDCS - Journal of Advanced Research in Dynamical and Control Systems - 2018. 07-Special Issue, pp. 1853-1859. http://jardcs.org/abstract.php?archiveid=5147.

29. Igor S. Konstantinov, G. S. Vasyliev, Oleg R. Kuzichkin, D.I. Surzhik, I. A. Kurilov, Sergey A. Lazarev. Development Of UV Communication Channels Characteristics Modeling Algorithm In A Mobile Ad-Hoc Network / Journal of Advanced Research in Dynamical and Control Systems (JARDCS) / ISSN: 1943-023X / Volume 11 | 08Special Issue, 2019. Pages: 1920-1928. http://www.jardcs.org/abstract.php?id=2543 